\title{
Effects of fatigue on the torque-velocity relation in muscle
}

\author{
O Spendiff, N T Longford, E M Winter
}

BrJ Sports Med 2002;36:431-435

See end of article for authors' affiliations

Correspondence to Dr Spendiff, 36 Sunholme Drive, Hadrian Lodge, Wallsend, Tyne \& Wear NE28 9YT,

owenspendiff@hotmail.com

Accepted 12 June 2002

\begin{abstract}
Background: The extent to which fatigue is related to the velocity of shortening is still not fully understood.

Objectives: To examine the effects of fatigue induced by maximal intensity exercise at different velocities on subsequent torque-velocity relations in muscle.

Methods: Ten men (mean (SD) age 25 (3) years; stature 1.78 (0.34) m; body mass 80.6 (14.5) kg) provided written informed consent and, over a five day period in a randomised manner, performed 16 fatiguing bouts of maximal intensity exercise on an isokinetic dynamometer each followed by one repetition maximum action. Concentric peak torque of the preferred leg was assessed at angular velocities of $0.52,1.05,2.09$, and $3.14 \mathrm{rad} / \mathrm{s}$. Mechanical work performed for all fatiguing bouts was held at 3050-3150 J. After a 45 second rest, a maximum effort was performed at one of the preset velocities. As a baseline, dependent variables of peak torque (N.m), angle of peak torque (rad), and mean torque (N.m) were measured at each velocity. An analysis of covariance was used to compare torque-velocity relations, and one factor, within subject analyses of variance with repeated measures investigated differences from baseline.

Results: Torque-velocity relations for all dependent variables did not differ ( $p>0.05)$. Similarly, angles at which peak torque occurred were not velocity dependent and did not differ from baseline and subsequent exercise measures ( $p>0.05)$. Generally, fatiguing exercise at low velocities led to reductions in peak and mean torque in subsequent exercise at higher velocities $(p<0.05)$.

Conclusions: Torque-velocity relations fundamentally remain intact after fatigue induced at any velocity, although the magnitude changes. The results suggest that the greater decline in torque during subsequent exercise at high velocities could be due to greater exhaustion of fatigue sensitive type II fibres, whereas low velocity subsequent exercise is less affected because of the greater use of type I fibres.
\end{abstract}

T he effect of fatigue on the kinetic behaviour of muscle has been the subject of long standing interest. ${ }^{1}$ Fatigue is traditionally defined as the failure to maintain a specific force or power output, and is characterised by a reversible decline in a muscle's force generating capacity that culminates in momentary failure. ${ }^{23}$ A suggested improvement to this definition is "a condition in which there is a loss in the capacity of a muscle for developing force and/or velocity, resulting from muscle activity under load and which is reversible by rest" ${ }^{4}$ This accounts for the impairment of force/torque and velocity in dynamic exercise. It has been identified that the maintenance of a high force/torque output is related to the economy of metabolic kinetics within the exercising muscle. ${ }^{5}$ This is dependent on the type and quality of muscle fibres recruited for the activity.

When there is an increase in exercise intensity, there is an increase in the number and type of muscle fibres recruited ${ }^{6}$ It has been further highlighted that, in very light exercise, the fatigue resistant slow twitch fibres contribute to force/torque output, but, as exercise intensity increases, the more fatigue sensitive fast twitch muscle fibres are recruited because they are capable of generating greater tension. ${ }^{7}$ In maximal intensity exercise (MIE), defined as "all out" activity, ${ }^{8}$ all muscle fibres are recruited to maximise force/torque production. ${ }^{9}$ Therefore, the type of muscle fibre recruited is dependent on the force/torque-velocity relations of the activity under scrutiny. However, in sport, the dynamic function of muscle is diverse and many sports require an athlete to execute specific muscle actions at a wide range of velocities with differing force/torque outputs and little rest between. In many cases, success is dependent on the ability to resist the effects of fatigue so that decrements in each subsequent bout of activity are minimised. ${ }^{10}$

Recent studies ${ }^{611}{ }^{12}$ have examined this phenomenon by investigating the effect of prior submaximal exercise at low and high velocities on subsequent power output. They hypothesised that, during cycling at low velocities, a greater contribution would be made by slow twitch fibres, whereas at high velocities a greater contribution would be made by the fast twitch fibres. The results indicate that a prior fatiguing bout at high pedalling frequency had a greater diminishing effect on power output than prior fatigue induced by a slow pedalling frequency. They also indicate that, during high velocity exercise, the recruitment capability of the slow twitch fibres might have been exceeded and thus only the fatigue sensitive fast twitch fibres were able to contribute to torque output. This had a greater diminishing effect on subsequent power output. Also, as higher velocity exercise elicits a greater number of muscle actions, there may be a greater consumption of ATP. ${ }^{612}$

This previous research does not take into account prior exercise of maximal intensity. As all fibre types are recruited during MIE, this means the fatigue sensitive fast twitch fibres would also be recruited during low velocity exercise. ${ }^{13}$ The effects of this on the torque-velocity relations of subsequent exercise are yet to be investigated. The fact that exercise is maximal may alter the fatigue-velocity relation observed in submaximal exercise. Another important consideration in velocity dependent relations of fatigue on torque output is the subsequent velocity at which the muscles are exercised after experiencing fatigue. It has been proposed that muscle fatigue is specific to the movement patterns of the exercise. ${ }^{3}$ As adaptations in torque production are specific to the velocity at which the muscles are trained ${ }^{14}$ and there is also a possibility of selective recruitment of motor units with increasing velocities in MIE, ${ }^{13}$ fatigue may also be specific to the velocity of action.

Although the torque-velocity relation in muscle has received much attention both in vitro and in vivo and has great 
significance in sport performance, the extent to which fatigue is related to the velocity of shortening is still not fully understood. The purpose of this study was to examine the effects of fatigue induced by maximal intensity exercise at different velocities on subsequent torque-velocity relations in muscle.

\section{METHODS}

Subjects

Ten men of mean (SD) age 25 (3) years, stature $1.78(0.34) \mathrm{m}$, and body mass 80.6 (14.5) kg volunteered, gave written informed consent, and participated.

\section{Equipment}

All procedures were institutionally approved.

\section{Isokinetic dynamometer}

All measurements of torque and velocity were made using a Chattecx Kin-Com (Active system, model 125E Plus; Chattanooga Group Inc, Hixson, Tennessee, USA). The apparatus allowed the quadriceps of each subject's favoured leg to be maximally exercised at preset velocities for a prescribed number of repetitions. Then one maximal effort was measured for peak torque (N.m), angle of peak torque (rad), and mean torque (N.m) for the same velocities. Equipment was calibrated, tested for reproducibility, and data processed through Kin-Com software on a 386 SX-33 MHZ touch screen microcomputer. Baseline and subsequent exercise values were measured using the overlay protocol, and the prior exercise bouts were performed using the continuous protocol.

\section{Dynamometer set up}

Testing was performed with participants sitting upright and with the lateral epicondyle of their knee aligned with the axis of the dynamometer's resistance lever. The force pad was placed in a comfortable position across the participant's tibia with the distance of the fulcrum recorded. The hips were stabilised by means of a seat belt while the thigh and ankle were supported by velcro strapping for stabilisation and isolation of the tested muscle group. All bouts of fatiguing and subsequent exercise were measured over $1.05 \mathrm{rad}$ of flexion. The start angle ( $1.57 \mathrm{rad}$ ) was positioned just beyond the vertical alignment of the lever arm so that the exercised leg was slightly extended. The stop angle (0.52 rad) was chosen as it allowed the quadriceps to be exercised to fatigue and still exert torque at the extreme range of motion. As the exercise involved moving the limb through a gravity dependent position, additional force was required to accelerate the limb. Correction for gravity was made by using the dynamometer's gravity correction procedure which accounted for the gravitational effects of the subject's limb and lever arm. Also, for reliability, the same gravity correction factor obtained during the initial test was used for each subsequent test. ${ }^{13}$

\section{Experimental procedure}

On their arrival at the laboratory, the stature and body mass of the participants were recorded; they were then fully accustomed to the dynamometer and the testing procedures. The experiment was conducted over a five day period, with days 2 and 4 as rest days. All participants were instructed to abstain from exercise during the five days of the experiment. On the first day, concentric peak torque of the preferred leg was assessed at angular velocities of 0.52, 1.05, 2.09, and $3.14 \mathrm{rad} / \mathrm{s}$, and peak torque (N.m), angle of peak torque (rad), and mean torque (N.m) for each velocity were recorded as baseline. Participants then performed, in a randomised manner, 16 fatiguing bouts of maximal intensity exercise, four bouts at each velocity and each followed (after a 45 second rest) by a one repetition maximum action at one of these velocities (an example of the procedure is shown in fig 1). To accomplish

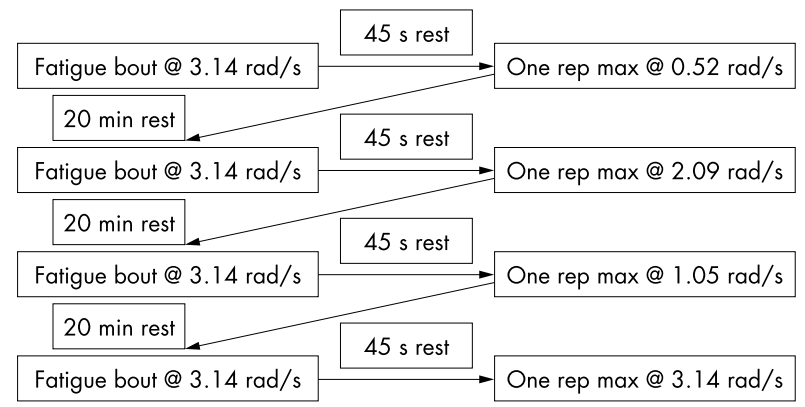

Figure 1 A schematic illustration of the experimental design for fatiguing exercises using $3.14 \mathrm{rad} / \mathrm{s}$ as an example. NB this figure is only for clarification of the experimental protocol. Sixteen bouts of fatiguing exercise (four at each velocity) were performed and each required a one repetition maximum after 45 seconds of rest. All bouts of exercise were randomised during testing.

this, five fatiguing bouts and their subsequent exercise measures were performed on day 1 after the pre-baseline measures were recorded, six on day 3 , and the five remaining bouts were performed on the fifth day. Post-baseline measures were also taken on the fifth day to identify possible adaptation.

Before each bout, participants warmed up by running on a treadmill (Powerjog, GXC200) for five minutes at a self selected speed. The speed selected on this initial occasion was used for each subsequent occasion. Before any measurements, five warm up repetitions were performed to accustom participants to the velocity at which they were about to exercise to induce fatigue. For each exercise bout, participants placed their hands on their hips for stabilisation and maximally exercised the quadriceps of their preferred leg in the concentric (shortening) mode of action. To address the stretchshorten cycle characteristic inherent in muscle, participants were instructed to produce torque in a smooth continuous action and not to kick back at the beginning phase of muscle action. The exercised limb was passively returned to the starting position at the same velocity to ensure that the muscle was exercised in only the concentric mode of action. To ensure maximum effort, the touch screen monitor was placed so that the subject could view a histogram illustration of peak torque output for each repetition, and verbal encouragement was given throughout. On completion of the fatiguing bout, the participant remained in position for 45 seconds and was then instructed to perform one maximal effort at one of the four velocities. A warm down was performed on the treadmill for five minutes after each bout of exercise, and a rest of 20 minutes was implemented between bouts to allow full recovery.

\section{Calculations of work}

An effort was made to equate the work rates of the prior exercise bouts so that a similar degree of fatigue was experienced at each velocity. From the equation

$\mathrm{s}=\frac{\mathrm{r} \pi \vartheta}{180}=0.017453293 \mathrm{r} \vartheta$

(where $\partial=$ the range of motion $\left(60^{\circ}\right), \mathrm{r}=$ the lever arm length (29-35 cm, dependent on subject leg length), s = the angular distance $(\operatorname{arc}))$, the distance of the angular motion of the leg multiplied by the mean torque of the prior baseline gave the work rate for one repetition. Fifteen repetitions were chosen at $0.52 \mathrm{rad} / \mathrm{s}$, and the number of repetitions for each of the three remaining velocities was calculated using the same equation. This was accomplished by multiplying the distance of angular motion by the corresponding baseline mean torque outputs for that velocity, then dividing this figure into the work rate for $0.52 \mathrm{rad} / \mathrm{s}$ to derive the number of repetitions for that velocity. 
Table 1 Summary of group mean (SEM) results of all exercise protocols on mean torque, peak torque, and angle of peak torque at 0.52 and $1.05 \mathrm{rad} / \mathrm{s}$

\begin{tabular}{|c|c|c|c|c|c|c|}
\hline & \multicolumn{3}{|l|}{0.52} & \multicolumn{3}{|l|}{1.05} \\
\hline & Mean torque (N.m) & Peak torque (N.m) & $\begin{array}{l}\text { Angle of peak torque } \\
\text { (rad) }\end{array}$ & Mean torque (N.m) & Peak torque (N.m) & $\begin{array}{l}\text { Angle of peak torque } \\
\text { (rad) }\end{array}$ \\
\hline Baseline & $255.84(15.41)$ & 314.42 (12.87) & $0.31(0.04)$ & $232.44(16.75)$ & $286.30(15.41)$ & $0.38(0.04)$ \\
\hline 0.52 & 202.42 (15.41) & 250.67 (11.99) & $0.37(0.06)$ & $178.20(15.52)$ & 218.04 (17.11) & $0.36(0.07)$ \\
\hline 1.05 & $222.00(15.13)$ & 274.15 (15.32) & $0.35(0.05)$ & 192.88 (13.36) & 236.77 (11.46) & $0.42(0.07)$ \\
\hline 2.09 & 225.76 (14.29) & 261.23 (15.04) & $0.32(0.05)$ & 198.87 (14.36) & 242.90 (12.43) & $0.34(0.06)$ \\
\hline 3.14 & 228.05 (13.35) & $263.08(13.26)$ & $0.31(0.05)$ & 204.71 (13.67) & 251.61 (13.17) & $0.38(0.07)$ \\
\hline Post-baseline & 256.39 (17.04) & 308.20 (18.08) & 0.39 (0.05) & 231.48 (18.80) & 280.83 (18.74) & 0.34 (0.05) \\
\hline
\end{tabular}

Table 2 Summary of group mean (SEM) results of all exercise protocols on mean torque, peak torque, and angle of peak torque at 2.09 and $3.14 \mathrm{rad} / \mathrm{s}$

\begin{tabular}{|c|c|c|c|c|c|c|}
\hline & \multicolumn{3}{|l|}{2.09} & \multicolumn{3}{|l|}{3.14} \\
\hline & Mean torque (N.m) & Peak torque (N.m) & $\begin{array}{l}\text { Angle of peak torque } \\
\text { (rad) }\end{array}$ & Mean torque (N.m) & Peak torque (N.m) & $\begin{array}{l}\text { Angle of peak torque } \\
\text { (rad) }\end{array}$ \\
\hline Baseline & $202.43(12.83)$ & $251.86(12.27)$ & $0.36(0.05)$ & $172.11(10.01)$ & $222.48(9.93)$ & $0.36(0.05)$ \\
\hline 0.52 & $152.08(12.51)$ & $191.68(12.36)$ & $0.44(0.05)$ & $130.01(8.61)$ & $177.79(10.92)$ & $0.44(0.10)$ \\
\hline 1.05 & 161.50 (11.88) & 200.51 (13.77) & $0.45(0.06)$ & 134.33 (7.92) & $182.03(9.48)$ & $0.50(0.11)$ \\
\hline 2.09 & 154.58 (12.37) & 203.71 (10.60) & $0.37(0.06)$ & 143.28 (9.29) & $196.46(10.40)$ & $0.56(0.12)$ \\
\hline 3.14 & $162.53(11.72)$ & $208.68(7.81)$ & $0.45(0.08)$ & $139.57(6.71)$ & $188.10(6.58)$ & $0.53(0.11)$ \\
\hline Post-baseline & 201.25 (15.19) & 251.18 (14.67) & $0.43(0.06)$ & $171.62(9.94)$ & $227.06(10.44)$ & $0.65(0.12)$ \\
\hline
\end{tabular}

Given that less torque was produced at higher velocities, more repetitions were performed with increases in velocity to ensure that the amount of work was similar between fatiguing bouts. The number of repetitions ranged from 15 at the low velocities to 26 at the highest velocity. As this method only allowed the amount of work to be estimated, for meaningful comparisons, mean mechanical work performed for all fatiguing bouts was held at 3050-3150 J.

\section{Statistical analysis}

Using pre-baseline as a covariate, an analysis of covariance was used to compare torque-velocity relations. Dependent variables were analysed by one factor, within subject analysis of variance with repeated measures. Related $t$ tests with Bonferroni correction identified where significant differences occurred. Significance was accepted at $\mathrm{p}<0.05$.

\section{RESULTS}

Tables 1 and 2 present a summary of group mean (SEM) results of the baselines and prior exercise bouts on peak torque (N.m), angle of peak torque (rad), and mean torque (N.m). Baseline and post-baselines did not differ $(p>0.05)$, which indicated that there were no appreciable learning effects or adaptation from participation. Accordingly, baseline measures were adopted for comparison with subsequent exercise measures. Also, torque-velocity relations for all dependent variables did not differ $(\mathrm{p}>0.05)$.

Figures 2-4 show comparisons of group mean results for peak torque (N.m), angle of peak torque (rad), and mean torque (N.m).

\section{Peak torque}

Peak torque (fig 2) in subsequent exercise was less than baseline at $0.52 \mathrm{rad} / \mathrm{s}$ after fatiguing exercise at $0.52 \mathrm{rad} / \mathrm{s}$ $(\mathrm{p}<0.05)$. The subsequent exercise at $1.05 \mathrm{rad} / \mathrm{s}$ led to reductions between baseline and fatiguing exercise at 0.52 and 1.05 $\mathrm{rad} / \mathrm{s}(\mathrm{p}<0.05)$. The subsequent exercise at $2.09 \mathrm{rad} / \mathrm{s}$ led to a greater reduction between baseline and all measures of fatigue $(\mathrm{p}<0.05)$, and subsequent exercise at $3.14 \mathrm{rad} / \mathrm{s}$ after

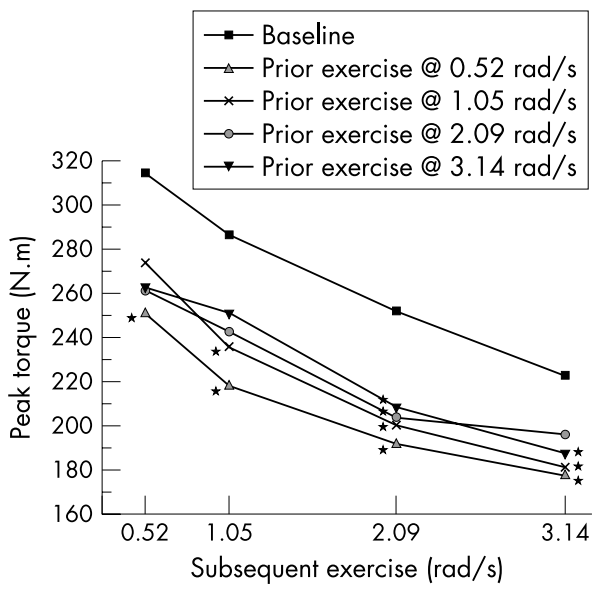

Figure 2 Group mean comparisons between peak torque baseline and the four subsequent peak torques after fatiguing exercise at the four set velocities. *Significant difference from baseline $(p<0.05)$.

fatiguing exercise at $0.52,1.05$, and $3.14 \mathrm{rad} / \mathrm{s}$ was also accompanied by reductions $(\mathrm{p}<0.05)$.

\section{Angle of peak torque}

Angles (fig 3) at which peak torque occurred were not velocity dependent and did not differ from baseline and subsequent exercise measures $(\mathrm{p}>0.05)$.

\section{Mean torque}

Mean torque (fig 4) in subsequent exercise at $0.52 \mathrm{rad} / \mathrm{s}$ after fatiguing exercise at 0.52 and $1.05 \mathrm{rad} / \mathrm{s}$ was less than baseline $(\mathrm{p}<0.05)$, and subsequent exercise at $1.05 \mathrm{rad} / \mathrm{s}$ led to reductions between baseline and fatiguing exercise at 0.52 and 1.05 $\mathrm{rad} / \mathrm{s}(\mathrm{p}<0.05)$. The subsequent exercise at 2.09 and $3.14 \mathrm{rad} / \mathrm{s}$ led to reductions between baseline and all fatiguing exercise measures $(\mathrm{p}<0.05)$. 


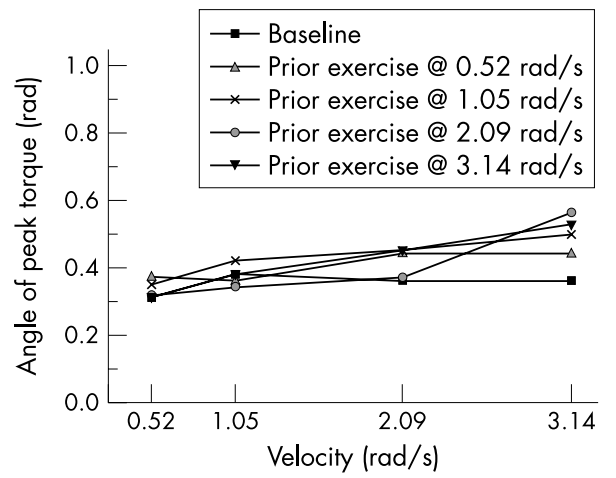

Figure 3 Group mean comparisons between angle of peak torque baseline and the four subsequent angles of peak torque after fatiguing exercise at the four set velocities.

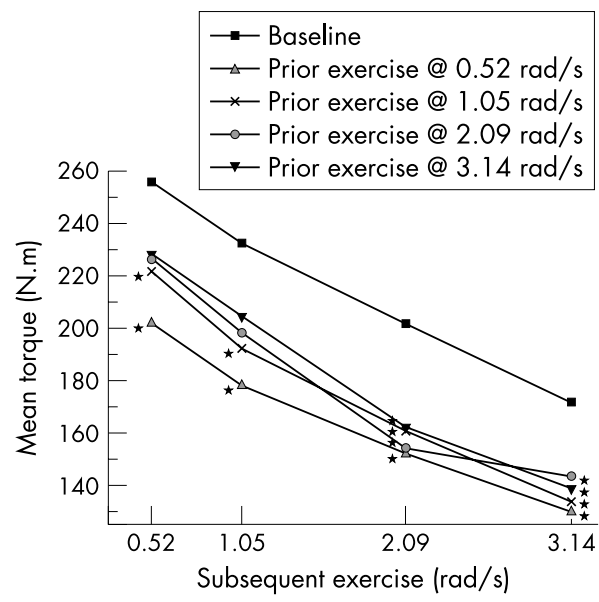

Figure 4 Group mean comparisons between mean torque baseline and the four subsequent mean torque measures after fatiguing exercise at the four set velocities. *Significant difference from baseline $(p<0.05)$.

\section{DISCUSSION}

The main findings of this study are that fundamentally torque-velocity relations remain intact after fatigue induced at any velocity, although the magnitude changes. Figures 2 and 4 illustrate the decline in peak torque and mean torque measures; both show a curvilinear trend with increments in velocity. The angle of peak torque (fig 3) did not change $(\mathrm{p}>0.05)$, although there was a slight trend for the angle to occur later in the range of motion as the velocity of exercise increased, which has been observed in previous research. ${ }^{15}$ Our results show that maximal intensity fatiguing exercise at low velocities elicits a greater reduction in torque in subsequent exercise at all velocities, with a pronounced decline in torque at higher velocities.

However, we used MIE, whereas previous research investigated fatigue induced by submaximal exercise. During submaximal exercise the relevant contribution of different fibre types was proposed to explain the observed velocity dependent effect of prior exercise on power output in cycling. ${ }^{6}{ }^{12}$ It was also hypothesised that the number of preceding muscle actions and increases in muscle temperature increases the rate of ATP consumption, ${ }^{611}{ }^{12}$ although no account was taken of possible differences in internal work performed. In a further study ${ }^{16}$ comparisons were made between the glycogen concentrations of the muscle fibres after submaximal exercise at 6.3 and $12.6 \mathrm{rad} / \mathrm{s}$, which showed that decruitment of the type I fibres does not occur at higher velocities, although the authors proposed that the type I fibres "are operating on the descending arm of a mechanical efficiency/velocity relationship". ${ }^{16}$ The depletion of glycogen in the fast twitch fibres was greater at the higher velocity and less at the lower velocity when compared with the type I fibres. The apparent velocity dependent effect of submaximal prior exercise on subsequent exercise proposed by these authors may have been suppressed by MIE used in this study. Moreover, the mechanisms responsible may operate differently when prior MIE induces fatigue over increasing velocities.

Indeed, it has been identified that, during submaximal exercise, both fibre types contribute to torque at low and high velocities, ${ }^{11}$ although the relative contributions favour slow twitch during low velocity exercise and fast twitch during high velocity exercise. In MIE, all fibre types are recruited during performance at all velocities, ${ }^{13}$ although, because of the greater number of cross bridge interactions at the lower velocities, more torque is produced. As the larger fast twitch fibres contain more protein filaments, they have greater torque generating potential. Also, as they use the anaerobic glycolysis system and have a more extensive sarcoplasmic reticulum, which in turn allows a more rapid release of calcium ions across the sarcolemma, they can generate torque more rapidly. ${ }^{17}$ Thus, during maximal exercise at all velocities the relative contribution of the fast twitch fibres is increased compared with exercise at the same velocity in submaximal exercise. This would influence the amount of fatigue experienced during maximal exercise at the lower velocities.

Research has also highlighted that the maximum shortening velocity of muscle is related to the rate at which the cross bridges cycle, irrespective of the number of cross bridges interacting and sarcomere length. ${ }^{17}$ Every actin-myosin interaction requires the hydrolysis of one molecule of ATP. ${ }^{18}$ As torque declines linearly with increases in the velocity of action, then as the velocity of exercise increases, less ATP is hydrolysed per repetition. As the type II fibres consume ATP at a greater rate and more is hydrolysed at the lower velocities, more metabolic acidosis will occur. This decreases sodium and calcium ion release, which in turn results in a decline in potassium concentration and ultimately restricts cross bridge kinetics. ${ }^{19}$ Therefore, as the fast twitch muscle fibres predominate for each velocity of prior exercise, fatigue during maximal exercise may be related to the amount of torque generated rather than the velocity at which the force is being generated.

It has been proposed that, during repetitive concentric exercise, the pattern of the firing frequencies can be altered. ${ }^{20}$ Indeed, research has indicated that slow twitch motor units fire continuously for long periods, whereas the fast twitch motor units fire at high rates, but only intermittently. ${ }^{20}$ In mixed muscle such as is typically observed in the human quadriceps, maximal torque is produced when both slow and fast twitch pools of motor units are recruited at an optimal rate coding to produce a fused tetanus synchronously. ${ }^{20}$ If one type of motor unit cannot initiate optimal delivery of nerve signals to muscle fibres, optimal recruitment of muscle fibre for maximal force production is diminished. ${ }^{20}$ Therefore, another reason why fatigue in MIE could be greater at lower velocities is that each repetition requires the activation of muscle fibres for a greater duration over the same range of motion. The experience of fatigue may cause greater disruption to the synchronous behaviour of the motor unit's firing frequencies throughout each repetition and from one repetition to the next.

A further possible reason why low velocity prior exercise causes greater decline in torque output is central fatigue. During the testing procedures, volunteers reported that a greater degree of psychological rather than muscular discomfort occurred at the lower velocities. Central fatigue is determined by a dysfunction within the neural pathways that send and receive command signals to and from the muscle. ${ }^{9}$ It has been proposed that central fatigue is caused by inhibition of the recruitment of motor areas as the result of nervous impulses 


\section{Take home message}

The finding that low velocity exercise reduces torque at high velocities more than at low velocities could be relevant to those who use isokinetic dynamometery for strength training, injury prevention, and rehabilitation.

from the chemoreceptors in the fatigued muscles. ${ }^{21}$ Central fatigue in maximal exercise may be caused by inhibition of voluntary effort because of the discomfort in the muscle. ${ }^{22}$ It should be noted that during the experimental protocol every participant was encouraged by vocal and visual (force histogram) stimuli for every repetition. Another possible limitation is the effect of breath holding (valsalva manoeuvre) during the fatiguing and subsequent maximal actions, which is difficult to control. This could increase muscle acidity during exercise, which would interfere with torque production, especially during the longer duration, low velocity exercise.

In this study, subsequent exercise was more diminished at the higher velocities after low and high velocity fatiguing bouts of exercise. The more decisive effect of fatigue at higher velocities on subsequent exercise is perhaps due to greater exhaustion of more muscle fibres. Specifically, the fatigue sensitive type II fibres at the onset of acidosis would interfere with kinetic behaviour of the muscles, thereby decreasing cross bridge detachments and reducing the ability of the cross bridges to cycle. ${ }^{6}$ This occurs because there is a limited supply of calcium, an inhibition of glycolytic regulatory enzymes such as phosphorylase and phosphofructokinase, and an increase in ADP. Therefore, subsequent torque output at the higher velocities would be more affected by fatigue induced at any velocity. ${ }^{7}$ However, it should be noted that, as most human movement involves a stretch-shorten cycle and we have only examined concentric isokinetic muscle actions, our findings are limited to isokinetic muscle activity.

To conclude, we have shown that, fundamentally, torquevelocity relations remain intact after fatigue induced at any velocity, although the magnitude changes. Maximal intensity fatiguing exercise at low velocities elicits a greater reduction in torque in subsequent exercise at all velocities, with a pronounced decline in torque at higher velocities. These results suggest that maximal intensity exercise at low velocity has a greater fatiguing effect than that induced by high velocity. The greater fatigue experienced during low velocity exercise could be attributable to recruitment of both type I and type II muscle fibres. This produces greater metabolic acidosis than higher velocities, during which mainly type II fibres are recruited, ${ }^{9}$ thus decreasing torque in subsequent exercise. The greater decline in torque during subsequent exercise at high velocities is perhaps due to greater exhaustion of more muscle fibres, specifically the fatigue sensitive type II fibres, whereas low velocity subsequent exercise is less affected because of greater use of the more fatigue resistant type I fibres.
Authors' affiliations

O Spendiff, Department of Sport, Health and Exercise, Staffordshire University, Stoke on Trent, UK

N T Longford, Department of Medical Statistics, De Montfort University, Leicester, UK

E M Winter, Centre for Sport and Exercise Science, Sheffield Hallam University, Sheffield, UK

\section{REFERENCES}

1 Hawley JA, Myburgh KH, Noakes TD, et al. Training techniques to improve fatigue resistance and enhance endurance performance. I Sports Sci 1997; 15:325-33.

2 Edwards RHT. Human muscle function and fatigue. In: Porter R, Whelan $J$, eds. Human muscle fatigue: physiological mechanisms London: Pitman Medical, 1981:1-18

3 McLester JR. Muscle contraction and fatigue. Sports Med 1997:23:287-305

4 Faulkner JA, Brooks SV. Fatigability of mouse muscles during constan length shortening, and lengthening contractions: interactions between fiber types and duty cycles. In Sargeant AJ, Kernell D, eds. Neuromuscular fatigue. Amsterdam: North Holland, 1993.

5 Karlsson J, Siödin B, Jacobs I. et al. Relevance of muscle fibre type to fatigue in short intense and prolonged exercise in man. In: Porter $R$, Whelan J, eds. Human muscle fatigue: physiological mechanisms. London: Pitman Medical, 1981:59-98.

6 Beelen A, Sargeant AJ. Effect of fatigue on maximal power output at different contraction velocities in humans. J Appl Physiol 1991:71:2332-7.

7 Green HJ. Mechanisms of muscle fatigue in intense exercise. J Sport Sci 1997; 15:247-56

8 Winter EM. Assessing exercise performance: maximal exercise I. British Journal of Physical Education Research Supplement 1991;9:12-14.

9 Edman KAP. Contractile performance of skeletal muscle fibres. In: Komi PV, ed. Strength and power in sport. Oxford: Blackwell Scientific Publications, 1992:96-114.

10 Williams C. Short term activity. In: Macleod RJ, Maughan M, Nimmo T, et al, eds. Exercise: benefits, limitations and adaptation. London: E \& FN Spon, 1983:59-62

11 Beelen A, Sargeant AJ. Effect of prior exercise at different pedalling frequencies on maximal power in humans. Eur J Appl Physiol 1993;66:102-7.

12 Cherry PW, Lakomy HKA, Nevill ME, et al. Effect of the number of preceeding muscle actions on subsequent peak power output. J Sport Sci 1997;15:201-6.

13 Perrin DH. Isokinetic exercise and assessment. Champaign, IL: Human Kinetics, 1993.

14 Coyle EF, Feiring DC, Rotkis TC, et al. Specificity of power improvements through slow and fast isokinetic training. J Appl Physiol 1981;51:1437-42.

15 Kannus $\mathbf{P}$, Beynnon B. Peak torque occurrence in the range of motion during isokinetic extension and flexion of the knee. Int J Sports Med 1993;14:422-6.

16 Beelen A, Sargeant AJ, Lind A, et al. Effect of contraction velocity on the pattern of glycogen depletion in human muscle fibre types. In: Sargeant AJ, Kernell D, eds. Neuromuscular fatigue. Amsterdam: Royal Netherlands Academy of Arts and Sciences, 1993:93-95.

17 Edman KAP, Reggiani C, Schiaffino S, et al. Maximum velocity of shortening related to myosin isoform composition in frog skeletal muscle fibres. J Physiol (Lond) 1988;395:679-94

18 Cheetham ME, Boobis LH, Brooks S, et al. Human muscle metabolism during sprint running. J Appl Physiol 1986;61:54-60.

19 McKenna MJ. The roles of ionic processes in muscular fatigue during intense exercise. Sports Med 1992;13:134-45.

20 Green HJ. Muscle power: fibre type recruitment, metabolism and fatigue. In: Jones NL, McCartney N, McComas AJ, eds. Human muscle power. Champaign, IL: Human Kinetics, 1986:65-80.

21 Assmussen E. Muscle fatigue. Med Sci Sports 1979;11:313-21.

22 Bannister EW. The perception of effort: an inductive approach. Eur J Appl Physiol 1979;41:141-50. 